\title{
Comment on 'Efficacy and toxicity of treatment with the anti-CTLA-4 antibody ipilimumab in patients with metastatic melanoma after prior anti-PD-1 therapy'
}

\author{
Keisuke Imafuku ${ }^{*}{ }^{1,2}$, Koji Yoshino ${ }^{1}$, Kei Yamaguchi ${ }^{1}$, Satoshi Tsuboi ${ }^{1}$, Kuniaki Ohara ${ }^{1}$ and Hiroo Hata ${ }^{2}$ \\ ${ }^{1}$ Department of Dermatology, Tokyo Metropolitan Cancer and Infectious Diseases Center, Komagome Hospital, Tokyo 113-8677, Japan and \\ ${ }^{2}$ Department of Dermatology, Hokkaido University Graduate School of Medicine, Sapporo 060-8638, Japan
}

Sir,

We carefully read the manuscript by Bowyer et al (2016) recently published in the British Journal of Cancer. They have retrospectively identified a cohort of 40 patients with metastatic melanomas who received anti-PD-1 monotherapy with pembrolizumab or nivolumab and who, after progression, were treated with ipilimumab.

The toxicity of ipilimumab was also evaluated in the report. Fourteen of the 40 patients demonstrated severe adverse events (over grade 3/4): nine with colitis, three with pneumonitis, two with hepatitis and one with encephalitis. Bowyer et al reported that two of the 40 patients simultaneously developed two severe immune-related adverse events (irAEs) (pneumonitis and hepatitis, diarrhoea and hepatitis). The median interval between the final dose of PD- 1 inhibitor and the initial administration of ipilimumab was 53 days (range 2-683 days).

At our institution (see Table 1), we administered ipilimumab as a second-line treatment for malignant melanoma to the 10 patients whose first-line nivolumab therapy had been unsuccessful. Six of the 10 patients had a short interval of less than 30 days (group A). Four of 10 patients had a long interval of more than 30 days (group B). All group A patients demonstrated severe adverse events (grade 3/4), with high fever of up to $40^{\circ} \mathrm{C}$. In addition, four patients simultaneously demonstrated two or more irAEs (one presented rash and colitis, colitis and hepatitis, rash and colitis, and rash and hepatitis). These four patients were unable to complete the course of ipilimumab because of the adverse events. In contrast, although the group B patients demonstrated irAEs without high fever, these were comparatively mild (not shown).

A similar report was published on PD-1 usage. Vemurafenib, a BRAF inhibitor for the treatment of malignant melanoma, is known to induce severe rash when it follows the administration of a PD- 1 inhibitor. When the interval between PD-1 and vemurafenib is less than 30 days, the adverse events tend to be more severe (Harding et al, 2012; Johnson et al, 2013; Imafuku et al, 2015).

We speculate that the difference is associated with the half-life in blood of nivolumab. The serum half-life of anti-PD-1 is 17-25 days. Therefore, it might be risky to administer ipilimumab within 30 days after nivolumab because of the high nivolumab concentration remaining in the serum.

The notable CheckMate 067 therapeutic trial of combined nivolumab and ipilimumab showed severe adverse events. Although the rate of monotherapy of nivolumab or ipilimumab induces, respectively, $5.1 \%$ and $13.2 \%$ adverse events over grade $3 / 4$, combined therapy causes $29.4 \%$ (Larkin et al, 2015). In addition, in the notable CheckMate 064 clinical trial of sequential administration of nivolumab and ipilimumab, the interval was 2 weeks. Adverse events of grade 3/4 tended to occur after the medication was switched (Weber et al, 2016).

According to these facts, in this report, we insist the risk of irAE is due to sequential usage of nivolumab and ipilimumab. When the interval is less than 30 days, two or more severe irAEs with high fever are likely to co-exist, as we experienced. Therefore, we have to correctly manage any irAEs that occur in the patients.

Since the efficacy of the combination or sequential therapy of nivolumab and ipilimumab is established, overcoming severe irAE is very essential when using immunotherapy. When the tumour activity is very high, we should start the next treatment. It is not always possible to leave a sufficiently long interval between the last dosage of nivolumab and the first of ipilimumab. It is important for physicians to understand the risks and benefits of sequential therapy and to keep quality of life in mind.

\section{CONFLICT OF INTEREST}

The authors declare no conflict of interest.

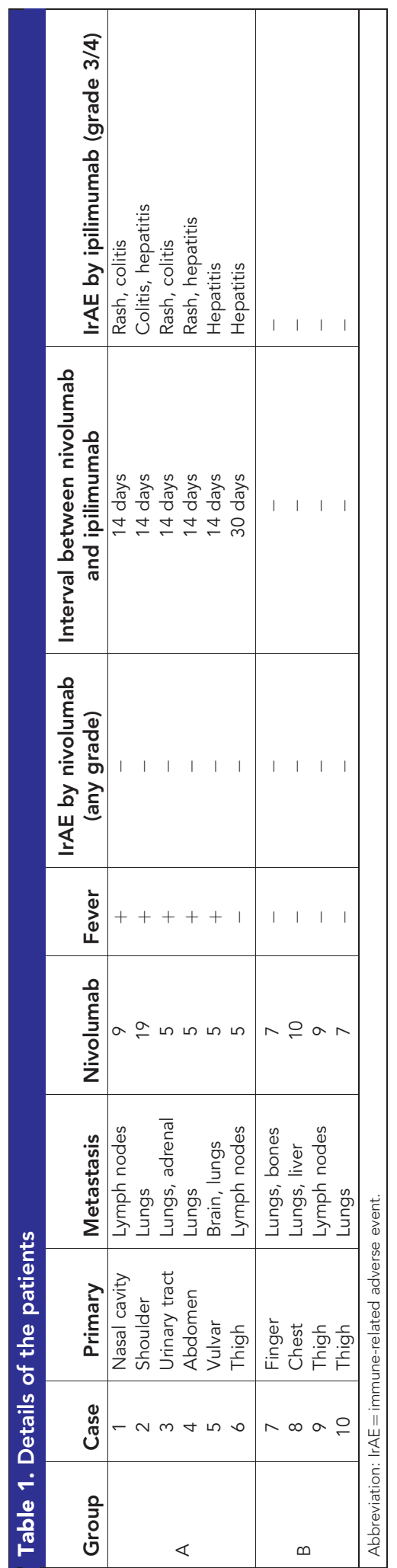




\section{REFERENCES}

Bowyer S, Prithviraj P, Lorigan P, Larkin J, McArthur G, Atkinson V, Millward M, Khou M, Diem S, Ramanujam S, Kong B, Liniker E, Guminski A, Parente P, Andrews MC, Parakh S, Cebon J, Long GV, Carlino MS, Klein O (2016) Efficacy and toxicity of treatment with the anti-CTLA-4 antibody ipilimumab in patients with metastatic melanoma after prior anti-PD-1 therapy. $\mathrm{Br} J$ Cancer 114(10): 1084-1089.

Harding JJ, Pulitzer M, Chapman PB (2012) Vemurafenib sensitivity skin reaction after ipilimumab. N Engl J Med 366(9): 866-868.

Imafuku K, Yoshino K, Ishiwata K, Otobe S, Tsuboi S, Ohara K, Hata H (2015) Severe rash associated with vemurafenib administration following nivolumab therapy. J Eur Acad Dermatol Venereol 30(10): e84-e86.

Johnson DB, Wallender EK, Cohen DN, Likhari SS, Zwerner JP, Powers JG, Shinn L, Kelley MC, Joseph RW, Sosman JA (2013) Severe cutaneous and neurologic toxicity in melanoma patients during vemurafenib administration following anti-PD-1 therapy. Cancer Immunol Res 1(6): 373-377.
Larkin J, Chiarion-Sileni V, Gonzalez R, Grob JJ, Cowey CL, Lao CD, Schadendorf D, Dummer R, Smylie M, Rutkowski P, Ferrucci PF, Hill A, Wagstaff J, Carlino MS, Haanen JB, Maio M, Marquez-Rodas I, McArthur GA, Ascierto PA, Long GV, Callahan MK, Postow MA, Grossmann K, Sznol M, Dreno B, Bastholt L, Yang A, Rollin LM, Horak C, Hodi FS, Wolchok JD (2015) Combined nivolumab and ipilimumab or monotherapy in untreated melanoma. N Engl J Med 373(1): 23-34.

Weber JS, Gibney G, Sullivan RJ, Sosman JA, Slingluff CL, Lawrence DP, Logan TF, Schuchter LM, Nair S, Fecher L, Buchbinder EI, Berghorn E, Ruisi M, Kong G, Jiang J, Horak C, Hodi FS (2016) Sequential administration of nivolumab and ipilimumab with a planned switch in patients with advanced melanoma (CheckMate 064): an open-label, randomised, phase 2 trial. Lancet Oncol 17(7): 943-955.

This work is published under the BJC's standard license to publish agreement. After 12 months the license terms will change to a Creative Commons AttributionNonCommercial-Share Alike 4.0 Unported License.

*Correspondence: Dr K Imafuku; E-mail: imafukukeisuke@gmail.com

Published online 21 March 2017

(C) 2017 Cancer Research UK. All rights reserved 0007-0920/17 